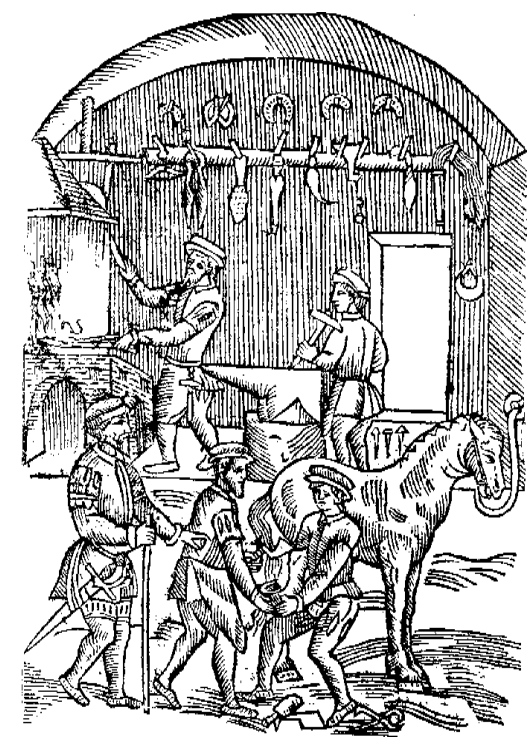

\title{
LA RESOLUCIÓN DE PROBLEMAS Y LA EDUCACIÓN EN CIENCIAS NATURALES
}

\author{
Margie N. Jessup \\ Profesora Asociada Universidad Pedagógica Nacional \\ Paulo Emilio Oviedo \\ Jefe Departamento de Posgrados U.P.N. \\ Rosalba P. de Castellanos \\ Profesora Asistente Universidad Pedagógica Nacional
}

Algunos resultados de la investigación educativa han permitido evidenciar diversos problemas en diferentes ámbitos, entre ellos el de la educación en ciencias naturales, tales como falta de interés de los estudiantes -incluidos los universitarios- hacia el trabajo en este campo a medida que avanzan en su formación (Raya, 1997), visión de la cien- cia y a la tecnología actuales como algo ajeno, impenetrable y totalmente fuera de su alcance (León, 1982) aprendizaje memorístico, carencia de autonomía y desarrollo progresivo de esquemas de pensamiento pobres, rígidos y estereotipados que conducen al estancamiento, a la rutina y a una elaboración intelectual superficial o de bajo nivel cognoscitivo (Amestoy de Sánchez, M. 1993) entre otros, que en nuestro medio aún tienen vigencia, a pesar de haber sido identificados tiempo atrás.

Lo anterior ha dado origen al desarrollo de múltiples investigaciones y modelos educativos, basados en diversas estrategias de cambio metodológico. Una de ellas ha 
sido la resolución de problemas, considerada por algunos como actividad fundamental de la ciencia y contrariamente por otros, como una expresión del pensamiento creativo y como proceso que incide en diversas esferas de la vida humana a niveles individual y social (Jessup, M. 1998). A su vez, esta última forma de asumir la resolución de problemas, ha permitido que en algunos enfoques de investigación en educación en ciencias naturales, así como en otras actividades educativas, laborales y cotidianas, se la tome como actividad central (Bransford - Stein. 1986; Garrett, 1988; Espíndola, J. L.1996). Además de lo anterior, la resolución de problemas constituye punto central de referencia para el trabajo en esferas como la inteligencia artificial, la evaluación de desarrollo de pensamiento complejo, de apropiación de habilidades y competencias, entre otros.

En el caso de las ciencias naturales, existen mayores desarrollos en cuanto a resolución de problemas en el campo de la física y de la química en comparación con la biología; de igual manera, la investigación educativa ha centrado fundamentalmente su atención en el nivel de educación básica y media, a di-

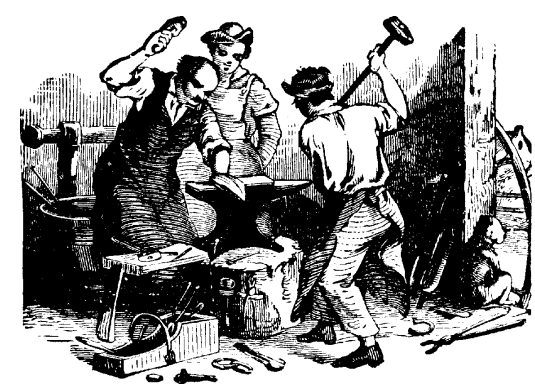

ferencia de la educación superior.

Esta investigación se centrará en la delimitación de los conceptos problema y resolución de problemas, así como en una aproximación a los tipos de problemas, en el marco de esta línea del Programa Interinstitucional de Doctorado en Educación, en el área de Ciencias Naturales.

\section{ACERCA DE LOS PROBLEMAS}

La línea de resolución de problemas como campo de investigación en el mundo, es muy activa y ha resultado de gran complejidad, especialmente en el último cuarto de siglo. Dicha complejidad está relacionada entre otros, con las concepciones de problema, resolución de problemas, enseñanza, aprendizaje, ciencia, investigación, educación y muchas más, subyacentes a las representaciones que sobre estos aspectos tienen las personas implicadas en los procesos de investigación, educación y otros; subsecuentemente, sus acciones están determinadas por tales representaciones. En otras palabras, las representaciones se manifiestan en los modos de obrar de tales personas.

Adicionalmente, los métodos usados por los educadores, psicólogos, filósofos y otros, para definir, describir o analizar y resolver problemas, determinan el método adoptado en la enseñanza, en la determinación de planes curriculares y en otras aplicaciones relacionadas con la educación (Greenfield, 1987).

Al considerar los anteriores aspectos, es posible comprender el

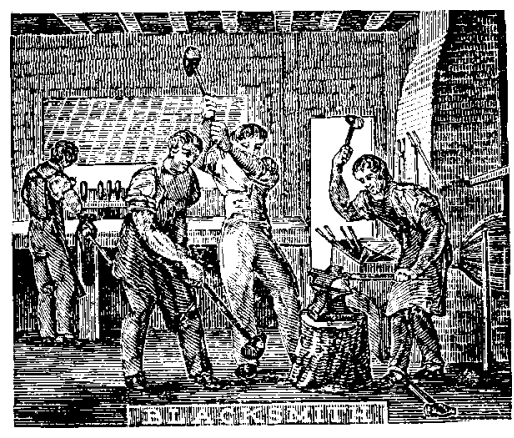

por qué de la riqueza de esta línea de investigación al igual que las causas de su complejidad, que dificulta la construcción de una única interpretación teórica acerca de los problemas y su resolución, así como también la estructuración de una visión sólida y general sobre el papel que juega la resolución de problemas tanto en investigación como en educación y otras esferas de la vida humana. De igual manera se posibilita inferir que dicha complejidad ha contribuido a determinar -para el caso de la investigación y la educación- la existencia de múltiples enfoques en dicho acercamiento, los cuales podrían ser tipíficados básicamente en tres: investigación y educación a) para b) sobre y e) centradas en la resolución de problemas (Gaulin, 1986).

De lo anterior se infiere la necesidad de teorizar sobre estos aspectos. En relación con ello, algunos investigadores señalan la necesidad de profundizar teóricamente en torno a qué constituye un problema en el ámbito de la resolución de problemas (Ramírez, 1990) en ciencias naturales, que se traduce en preguntas como: ¿Qué significa el término problema en el marco de la enseñanza de las ciencias? (Sigüenza-Sáez, 1990) ¿Existen 
tipos distintos de problemas? ¿Todos los procesos de resolución son iguales? ¿Hay tipos diferentes de resolventes? ¿Los problemas son fijos e independientes de su contexto y del resolvente? (Garrett, 1988) ¿Hasta dónde el problema de enseñar a resolver problemas se convierte en un problema de aprendizaje? (Cobo Lozano, 1996).

Por consiguiente, como referente inicial para la conceptualización y el análisis sobre este campo de investigación, es necesario establecer el significado que se asigna al término problema. Diversos autores consideran un problema como una situación que presenta dificultades para las cuales no hay soluciones evidentes (Hudgins, 1966; Riche, 1978; Mettes et al, 1980; Gil y Martínez, Torregrosa, 1983; Bodner y McMillen, 1986). En el mismo sentido y Krulik y Rudnik (1980) Tantean que "un problema es una situación, cuantitativa o no, que pide una solución, para lo cual los individuos implicados no conocen medios o caminos evidentes para obtenerla".

Aunque existen numerosas aproximaciones a la definición del concepto "problema", parece haber un cierto consenso en tomo a algunos aspectos relativos al mismo como son:

- Es una situación enigmática, espontánea o prevista, que difiere de una situación o meta deseada.

- Para dicha situación, no hay respuesta adecuada y eficaz inmediata.

- Si hay soluciones, no son evidentes.
- La situación puede ser considerada carencia pero también potencialidad, en tanto moviliza una conducta tendiente a la búsqueda de solución.

- Produce incertidumbre.

- Se presenta generalmente en forma de pregunta.

- El considerar dicha situación como problema o no, es algo estrictamente personal.

- Se presentan en diversos ámbitos de la vida humana.

- Su carácter está determinado por su formulación y por las etapas que implica el proceso de resolución.

- Su resolución implica creatividad.

Teniendo en cuenta lo anterior y con base en aspectos desarrollos relativos al tema, se podría tratar de hacer una aproximación a la definición del concepto, lo suficientemente general como para ser considerada válida en diversas esferas de la vida humana, en los siguientes términos:

Por problema se entiende una situación enigmática, espontánea o prevista, para la cual no se tiene una solución eficaz adecuada de manera inmediata, hecho que si bien produce incertidumbre y se convierte en una potencialidad para resolvería, dado que moviliza a quien la enfrenta hacia la búsqueda de solución. Su carácter de problema está dado fundamentalmente por la posibilidad de resolverlo o solucionarlo según los desarrollos del resolvente, así como por el proceso que implica su resolución.

Esta aproximación podría ser válida tanto para una situación relacionada con el campo de las ciencias naturales o sociales, la educación, la inteligencia artificial, la administración y la vida cotidiana.

Por otra parte, si se considera como problema una situación que presenta dificultades para las cuales no hay soluciones evidentes de manera inmediata, que por tanto produce cierta incertidumbre, cabe tener en cuenta dos aspectos: a) la(s) persona(s) que la enfrenta(n) y b) el contexto sociocultural e histórico en el cual se evidencia.

La conciencia de la situación y las consecuencias sobre las acciones que deriven de ella, es connatural a la persona, como único ser con capacidad para transformar conscientemente su entorno y desarrollar procesos de interacción social. Por ello, la reflexión acerca de la situación es fundamental, pues de ella depende el paso de la imprecisión a la determinación del(los) interrogante(s) que aclaran el (los) punto(s) objeto de atención.

Adicionalmente, es preciso tener en cuenta las individualidades, pues a partir de ellas -conocimientos, experiencias, tipo de inteligencia, creatividad...- se determinan en gran medida el abordaje y la reflexión sobre la situación dada y por consiguiente, el curso de acción a seguir frente al obstáculo que enfrenta la persona o el grupo que lo aborda.

Muy en relación con tales características, se ha desarrollado la idea de "Umbral de Problematicidad", diferente para cada persona, por encima del cual se puede decir que una situación, constituye un verdadero proble- 
ma para la(s) persona(s) en cuestión (Elshout, 1985).

En relación con uno de los puntos planteados como de consenso relativo, cabe pensar si toda pregunta es un problema o por el contrario, además del interrogante, se requiere la mediación de un proceso de búsqueda "sobre la naturaleza de la dificultad, para afrontar su resolución". Según diversos autores (Gil, D. 1987), sólo en el último caso se trata de problemas verdaderos, cuya delimitación requiere un proceso de elaboración a partir de la situación en que se suscita el interrogante.

Es posible plantear que aquellos interrogantes para cuya solución sólo se requieren cuestiones evidentes o la simple aplicación de procedimientos estandarizados, sin necesidad de un análisis contextual ni elaboración de relaciones entre los diferentes elementos que permiten explicar la situación, con el fin de abordarlos, constituyen pseudo problemas o ejercicios (de lápiz y papel, prácticas para confirmar resultados, entre otros).

Las precisiones expresadas sobre los verdaderos problemas y su diferencia con otras actividades que pueden relacionarse con ellos, son relevantes al tratar sobre la manera de abordarlos. Con apego a la definición propuesta, los problemas no se abordan simplemente mediante un protocolo que dé cuenta de ellos, que al ser aplicado genere la solución esperada; por el contrario, a partir de la situación será necesario desarrollar procesos de análisis (los cuales como se anotó anteriormente tie- nen las particularidades propias de quien los asume y por consiguiente, pueden variar de un individuo a otro), que permitan llegar a la formulación de la pregunta correspondiente.

Adicionalmente, como parte de la dinámica humana individual y social, en su diversidad se puede plantear la existencia de diferentes tipos de problemas cuya complejidad y viabilidad de resolución varían, según los referentes e implicaciones que están involucrados en la delimitación de los cuestionamientos derivados de la situación.

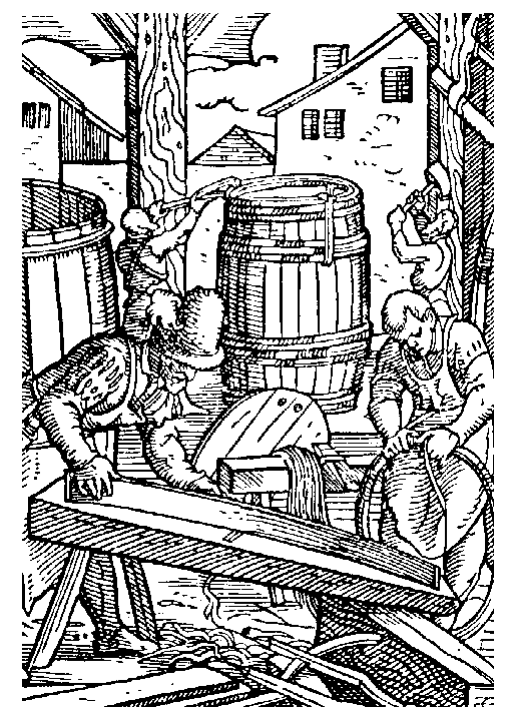

\section{TIPOS DE PROBLEMAS}

Al igual que existen diversas explicaciones que el ser humano, ha construido para explicarse el mundo, también en el campo de las situaciones que le generan dificultades hay diferentes ámbitos en los cuales se presentan problemas. Así por ejemplo, en la cotidianidad el tipo de situaciones que se deben afrontar corresponde a un grado de reflexión que puede ser diferente a aquél que resulta de la actividad intelectual o de la aplicación práctica profesional.

En la literatura es posible hallar diversas clasificaciones de problemas, todas ellas válidas atendiendo a los criterios que les subyacen, tales como: a) Campo de conocimiento implicado; b) tipo de tarea; c) naturaleza de enunciado y características del proceso de resolución (Perales Palacios, 1993).

Según el campo de conocimiento implicado, los problemas se podrían clasificar en: Científicos: pertenecen al ámbito de las ciencias; en ellos al parecer resulta más importante el proceso de resolución, que la obtención de la misma. No científicos. Usualmente forman parte de la vida cotidiana; en ellos resulta importante obtener soluciones de tipo práctico.

Para efectos prácticos, en este trabajo se hace énfasis en los problemas científicos y de manera particular, en el campo de la educación en ciencias naturales. Desde este punto de vista, si se tiene en cuenta el tipo de tarea o criterio de clasificación, éstos son de dos tipos: Cualitativos. Su resolución implica formas verbales/escritas, omitiendo las aproximaciones numéricas; se refieren a la interpretación científica de fenómenos reales y se les denomina frecuentemente "cuestiones". Cuantitativos. Exigen aproximaciones de orden numérico. 
Para el caso de la biología resulta importante tener en cuenta esta clasificación, por cuanto a diferencia de la física y de la química, no siempre los problemas pueden ser resueltos con cálculos numéricos.

Si se parte de la naturaleza del enunciado y de las características de su resolución podría decirse que existen varias clasificaciones, pues aunque todas contemplan como elementos básicos dos tipos de problemas, los abiertos y los cerrados, la forma como se asumen estos dos tipos presenta profundas diferencias, a saber:

Según López (1989), éstos se pueden caracterizar como: $\mathrm{Ce}$ rrados - contienen toda la información precisa y se resuelven mediante el empleo de un cierto algoritmo por parte del resolvente. Abiertos - se resuelven mediante la aplicación por parte, del resolvente, de una o varias etapas, mediante una acción de pensamiento productivo.

De acuerdo con los planteamientos de Perales (1993), los problemas cuantitativos podrían asumirse como cerrados, mientras que los cualitativos serían considerados abiertos.

Garrett (1988) por su parte, los asume de la siguiente manera: Rompecabezas - cuando pueden ser resueltos potencialmente dentro de un paradigma. Estas situaciones a su vez pueden ser: Cerradas - cuando para ellas puede haber una o más respuestas, pero todas igualmente correctas, o Abiertas - aquellas para las cuales puede haber varias respuestas, sin que ninguna sea correcta o errónea en términos absolutos, sino simplemente la más adecuada para un conjunto dado de circunstancias.

Problemas Verdaderos - son situaciones enigmáticas, no solubles ni resolubles, sino sólo comprensibles.

Desde el punto de vista de Frazer (1982), existen dos tipos de problemas: Artificiales - pueden tener o no un objetivo dirigido y a su vez pueden ser: Cerrados - si poseen una única solución, o Abiertos - si poseen un número variable de soluciones. En este tipo de problemas, la solución es conocida por quien los plantea. Reales - cuando no tienen solución, o no se les conoce.

Según lo anterior, se podría pensar en dos situaciones relacionadas con esta clasificación: a) que en el campo ¿educativo, frecuentemente se utilizan más problemas artificiales que reales; b) que de acuerdo con los desarrollos científicos actuales, un problema real en el campo de las ciencias naturales y particularmente de la biología no debería ubicarse necesariamente sólo en el marco de la disciplina, aun cuando presente un fuerte componente biológico; por consiguiente, su solución debería darse desde un acercamiento interdisciplinario o aún más, transdisciplinario.

Por último, valdría la pena plantearse un nuevo criterio de clasificación, como es el tipo de aproximación para la resolución. Acogiéndose a este criterio, bien podría plantearse una nueva clasificación:

Teóricos - cuando su resolución no implica aproximaciones de corte experimental. Experimentales- cuando su resolución implica aproximaciones de este tipo.

En este sentido sin embargo, resulta importante tener en cuenta que desde el punto de vista del planteamiento no existiría ninguna diferencia entre estos dos tipos de problemas puesto que el problema experimental lleva en sí mismo el planteamiento teórico adecuado.

Para concluir, sería importante destacar que el tipo de problemas considerado como reales por Frazer corresponde al de problemas verdaderos de Garrett, pero aparentemente se podría establecer un ligero matiz de diferencia entre los planteamientos de estos dos autores con relación a Perales, en lo atinente a los problemas abiertos y cerrados. No obstante, en todos los casos, el problema abierto sólo se resuelve, no se soluciona.

Este último planteamiento introduce entonces una cuestión de orden epistemológico, relacionada con el proceso de resolución de problemas propiamente dicho. Por ello, a continuación se tratará de profundizar en este aspecto.

\section{ACERCA DE LA RESOLUCIÓN DE PROBLEMAS}

En la literatura se encuentra un buen número de reportes sobre trabajos en los cuales se han empleado muchos métodos de análisis para estudiar la resolución de problemas en niños y jóvenes; tipos psicológicos y estilos cognitivos; simulación en computadora y procesamiento de información; análisis del pro- 
ceso de resolución propiamente dicho entre otros Greenfiel (1987).

En ellos, es Posible reconocer diferentes paradigmas sobre resolución de problemas; si se asumen éstos como formas diferentes de representarse este proceso y de entender su rol en el proceso de enseñanza y aprendizaje, es posible comprender que al igual que en el caso de los problemas, a estas representaciones subyacen ciertas concepciones y que de igual manera, tales representaciones se manifiestan en el modo de proceder las personas que resuelven los problemas.

De acuerdo con los planteamientos de Marín (1996), existen los paradigmas teoricista, tecnicista, modernista, constructivista, procedimental y de modelización, que de manera general pueden ser agrupados en dos grandes tendencias, según el centro fundamental de atención: a) bien en la resolución formal de los problemas, bien en la enseñanza de los mismos; b) en el solucionador de problemas.

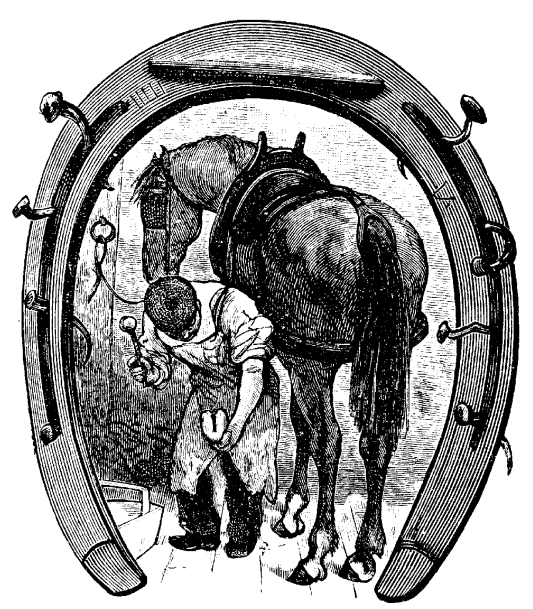

Para los propósitos de este escrito, la mirada se detendrá en el aspecto de la resolución de los problemas, que naturalmente involucro al resolvente. En este sentido, parece haber también un cierto consenso en tomo a los términos resolvery solucionar un problema.

Numerosos autores se refieren en diferentes términos a la solución y resolución de los problemas. Probablemente quien más explícitamente expone la diferencia entre ellos es Garrett (1988). Refiriéndose a ello, el autor plantea que se soluciona una situación cuando se obtiene una respuesta, mientras que se resuel$v e$, cuando no es posible obtener una solución definida-como es el caso de las situaciones abiertas. Por ello plantea que es más afortunado referirse a enfrentarse a un problema, que a solucionarlo.

Esta situación tiene relevancia, pues si los verdaderos problemas en el ámbito de las ciencias naturales tuviesen solución, se podría inferir que en algún momento sería posible contar con un conocimiento acabado y de esta forma se contribuiría a fomentar una visión de ciencia como un conjunto de "verdades" absolutas, fijas, obtenidas en un momento dado. En lugar de ello, el pensar en la posibilidad de resolver problemas, plantea así mismo la potencialidad de revaluar y contribuir con nuevas aproximaciones al desarrollo de conocimiento cualitativamente cada vez más complejo, que en última instancia no es otra cosa que el conjunto de las mejores explicaciones, concordantes con el desarrollo científico, tecnoló- gico, social y cultural de la humanidad.

Gran número de reportes se asemejan -en cuanto al proceso de resolución de los problemas- en los siguientes puntos fundamentales:

- Se consideran diferentes etapas que contemplan: identificación, delimitación o determinación del problema, exploración de estrategias de solución, acción con base en ellas, evaluación de los logros obtenidos.

- Se piensa que la resolución implica un proceso de pensamiento creativo (o productivo).

- Se asume que este proceso implica la utilización de desarrollos previos (individuales) del resolvente, así como la utilización por su parte de determinados procedimientos.

- Se tienen en cuenta -en términos generales- las condiciones propias de la persona que resuelve el problema.

- Se acepta que se utilizan diferentes tipos de memoria en el proceso de resolución.

Novak por su parte $(1982 ; 1988)$ considera, que la resolución de un problema implica así mismo la reorganización de la información almacenada en la estructura cognoscitiva de la persona que no resuelve, es decir, que hay aprendizaje significativo. En sentido similar, plantea que habrá aprendizaje significativo cuando el material aprendido encaja en la red cognoscitiva ya existente en quien aprende cuando probablemente el trabajo se realiza en la denominada por él zona de interés óptimo (que probablemente corresponde al ya mencio- 
nado umbral de dificultad). Esta zona sería intermedia entre la de información conocida y la de desconocimiento total; por consiguiente, constituye un límite personal.

En esos términos, la resolución de problemas podría ser considerada como un proceso mediante el cual, una persona que se enfrenta a un problema, trata de identificarlo, de delimitarlo, de explorar posibilidades de resolverlo, de elegir las estrategias adecuadas para lograrlo a partir de sus desarrollos individuales, de llevarlas a la práctica mediante la aplicación de métodos y técnicas apropiados.

Para el caso de la enseñanza de las ciencias naturales, han sido propuestos diversos modelos basados en la resolución de problemas, determinados por las representaciones que sobre este proceso tienen los proponentes. No obstante según los planteamientos de algunos autores, a pesar de la riqueza en cuanto a investigación y propuestas de formación centradas en esta estrategia de cambio metodológico, no es posible evidenciar resultados positivos en cuanto a aprendizaje significativo de las ciencias se refiere, Por ello, vale la pena cuestionarse sobre el origen de tal estancamiento.

\section{CONCLUSIONES}

Asumiendo como reales los planteamientos de quienes afirman que no hay un cambio significativo en términos de aprendizaje, a pesar de las numerosas investigaciones y modelos propuestos centrados en la resolución de problemas, tiene relevancia preguntarse acerca de la justificación de investigar y experimentar en este campo.

En otras palabras, el acercamiento a los procesos de indagación científica mediante la resolución de problemas, con el consiguiente desarrollo de habilidades básicas, procedimentales e investigativas, puede constituirse en una alternativa eficaz para incrementar la creatividad y la capacidad crítica de los estudiantes, lo cual se constituye a su vez en fundamento de la autonomía y responsabilidad social.

A partir de esta conceptualización es posible deducir la importancia de la resolución de problemas en el campo de la educación en ciencias naturales, partiendo de la asunción de la ciencia como una actividad social en permanente proceso de construcción y reconstrucción, con implicaciones en el desarrollo social y cultural de los pueblos y por tanto con un compromiso ético que le es inherente.

En este sentido y retornando los planteamientos de diversos autores, son varios los aspectos por los cuales amerita educar con base en este acercamiento; sin embargo, probablemente el más destacable entre ellos, es que este tipo de aproximación educativa permite al estudiante ser gestor de su propio aprendizaje, puesto que se enfrenta a una situación dada a partir de sus propios intereses expectativas, y teniendo como elemento fundamental para resolvería sus características individuales, logra la(s) mejor(es) respuesta(s) para la misma, desarrollando así mismo habilidades que le serán de utilidad en cualquier actividad de su vida, dado que el enfrentarse a problemas es una actividad cotidiana para el ser humano.

\section{BIBLIOGRAFÍA}

Amestoy de SÁnchez, Margarita. 1993. El desarrollo de habilidades de pensamiento y su aplicación a la enseñanza. Innovación en la educación universitaria en América Latina. OEA. Proyecto Multiregional de Educación Media y Superior PROMESUP. Programa Regional de Desarrollo Educativo PREDE, p. 139249

Bodner y McMillen. 1986 Cognitive Reestructuping as an Eariy Stage in Problem Solving. Journal of Rescarch in Science teaching. 23 (8), 727-737. Citado por: Ramírez Castro Juan Lorenzo. La resolución de problemas de física y química como investigación en la enseñan-

za media: un instrumento de cambio metodológico. Tesis doctoral. Universidad Autónoma de Barcelona. Fac. de Ciencias Químicas. 1990.

Bransford, J. D. Stein, B. S. 1986. Solución ideal de problemas. Ed. Labor.

Cobo Lozano, 1996. Análisis de las actuaciones de alumnos de $3^{\prime} d B$ UP en la Resolución de Problemas que comparan áreas de figuras geométricas. Enseñanza de las Ciencias, 14(2), 195-207.

Elshout, 1985. Citado por: Ramírez Castro Juan Lorenzo. La resolución de problemas de física y química como investigación en la enseñan- 
za media: un instrumento de cambio metodológico. Tesis doctoral. Universidad Autónoma de Barcelona. Fac. de Ciencias Químicas. 1990.

Espíndola, J. L. 1996. Análisis de problemas y toma de decisiones. Ed. Alambra Mexícana.

Frazer, M. J.: 1982. Solving Chemical Problems. Chemical Society Revíew, (2), 171-190.

Garrett. R. M. 1986. Issues in Science Education: Problema Solving Creativity and Originality. Intermational Journal Science Education, 9(2), 125-190.

GaRRETt, R. M. 1988. Resolución de problemas y creatividad: implicación para el currículo de ciencias. Enseñanza de las Ciencias, 6(3), 224230.

Gaulin, C. 1986. Tendencias actuales en la enseñanza de las matemáticas. (14), 11-18.

GIL, D. 1987. Los errores conceptuales como otigen de un nuevo modelo didáctico: de la búsqueda a la Investigación. Investigación en la escuela. 1, 35-42.

GiL D. y Martínez-TorRegrosa, Joaquín. 1983. Tres paradigmas básicos en la enseñanza de las ciencias. Enseñanza de las Ciencias. 1, 26-33.

GreenfieLd, L. B. 1987. Teaching Thinking Through Problem Solving. New Directions for Teaching and Leanúng. (30), 5-21.

HAYES, J. R. 1981. The Complete Problem Solver. Philadelphia, The Franklin Institute Press. Citado por: Ramírez Castro Juan Lorenzo. La resolución de problemas de física y química como investigación en la enseñanza media: un instrumento de cambio metodológico. Tesis Doctoral. Universidad Autónoma de Barcelona. Fac. de Ciencias Químicas. 1990.

Hudgins, B. B. 1966 . Cómo enseñar a resolver problemas en el aula. BuenosAires: Paidós.

JESSUP, M. 1998. Resolución de problemas y enseñanza de las ciencias naturales. TEA. Revista de la Facultad de Ciencia y Tecnología, (3), pp. 41-52.

León, A. I.; SolÉ, M. 1982. ¿Enseñamos realmente a investigar la naturaleza? Educación, México. No $42,167-186$.

LóPEZ, F. 1989. Dependencia - independencia de campo y educación científico. Revista de Educación, 289, 235-258.

Marín, N. 1996. Condiciones fundamentadas de enseñanza-aprendizaje para la resolución de problemas en ciencias. Seminario Preparatorio Doctorado en Educación Área Ciencias Naturales, Universidad Pedagógica Nacional. Santafé de Bogotá. Multilit. $13 \mathrm{p}$.

Mettes et al, 1980. Teaching and Learning Problem Solving in Science Part 1. A general Strategy. J. Chem. Ed. V. 57 pp. 882-885.

Novak, J. D. 1982. Teoría y práctica de la educación. Alianza Editorial. Madrid.

\footnotetext{
1988. Constructivismo humano: un consenso emergente. Enseñanza de las Ciencias, 14(1), 45-61.
}

Perales P., F. J., 1993. La resolución de problemas: una revisión estructurada. Enseñanza de las Ciencias, 11(2), 170-178.

Ramírez Castro, Juan Lorenzo. 1990. La resolución de problemas de física y química como investigación en la enseñanza media. un instrumento de cambio metodológico. Tesis Doctoral. Universidad Autónoma de Barcelona. Fac. de Ciencias Químicas.

Raya, A. 1997. Problemas sociales de la ciencia. Educación (La Habana), 34.

RICHE, N. 1978, Trame pour rédiger un exereise de physique. Revue Frangaise de Pédagogie. 45, 183199. Citado por: Ramírez-Castro Juan Lorenzo. La resolución de problemas de física y química como investigación en la enseñanza media: un instrumento de cambio metodológico. Tesis Doctoral. Universidad Autónoma de Barcelona. Fac. de Ciencias Químicas. 1990.

SigüEnZA, A. F.; SÁEZ, M. J. 1990, Análisis de la resolución de problemas como estrategia de la enseñanza de la Biología. Enseñanza de las Ciencias, 8(3), 223-230

Woods, D. R.; Crowe, C. M. y coautores. 1985. Challengs to Teaching Problem-Solving Skiiss. Chem. 13 News (Waterloo University), 15 5, 1-12. 